\title{
HOW A MINIMAL SURFACE LEAVES AN OBSTACLE ${ }^{1}$
}

\author{
BY DAVID KINDERLEHRER
}

Communicated by Hans Weinberger, May 5, 1972

\begin{abstract}
We announce that the function of least area among all functions defined in a convex domain, vanishing on its boundary, and constrained to lie above a concave analytic obstacle leaves the obstacle along an analytic curve.
\end{abstract}

We announce a result about the curve of separation determined by the solution to a variational inequality. A strictly convex domain $\Omega$ with smooth boundary $\partial \Omega$ is given in the $z=x_{1}+i x_{2}$ plane together with a smooth function $\psi(z)$ which assumes a positive maximum in $\Omega$ and is negative on $\partial \Omega$. Let $K$ denote the closed convex set of Lipschitz functions $v$ satisfying $v \geqq \psi$ in $\Omega$ and $v=0$ on $\partial \Omega$. Let us denote by $u$ the function of $K$ which minimizes area among all functions of $K$; that is

$$
u \in K: \quad \int_{\Omega} \frac{u_{x_{j}}}{\left(1+\left|u_{x}\right|^{2}\right)^{1 / 2}}(v-u)_{x_{j}} d x \geqq 0, \quad v \in K .
$$

The existence of such $u$, actually satisfying $u \in H^{2, q}(\Omega) \cap C^{1, \lambda}(\bar{\Omega})$, $1 \leqq q<\infty, \quad 0<\lambda<1$, was shown in the work of $H$. Lewy and G. Stampacchia [7] and also in M. Giaquinta and L. Pepe [1]. For $u$ there is a set of coincidence $I$ consisting of the points $z \in \Omega$ where $u(z)=\psi(z)$. Let us call

$$
\Gamma(u)=\Gamma=\left\{\left(x_{1}, x_{2}, x_{3}\right): x_{3}=u(z)=\psi(z), z \in \partial I\right\}
$$

the "curve" of separation.

Up to this time it has only been known that when $\psi$ is smooth and strictly concave, $\Gamma$ is a Jordan curve [2]. On the other hand, the corresponding problem for the $u \in K$ minimizing the Dirichlet integral has been thoroughly studied by $\mathrm{H}$. Lewy and G. Stampacchia [6]. We wish to announce here the

THEOREM. Let $\psi$ be analytic and strictly concave. Let $u$ be the solution of (1). Then $\Gamma(u)$ is an analytic Jordan curve (as a function of its arc length parameter).

The demonstration relies on the resolution of a system of differential equations and the utilization of the system to extend analytically a con-

AMS 1970 subject classifications. Primary 35J20; Secondary 53A10.

Key words and phrases. Minimal surface, variational inequality, rectification of curves, analytic extension.

${ }^{1}$ This research was supported by ASOFR 71-2098; AFOSR 883-67 and the Consiglio Nazionale delle Richerche. 
formal representation of the minimal surface which is the graph of $u$ in the subset of $\Omega$ where $u>\psi$. This is the idea of Hans Lewy (cf. for example [4], [5]). To derive the system of equations requires knowing that $u$ has bounded second derivatives, which was shown in [3]. In order to identify the solution, we prove first that $\Gamma$ is rectifiable.

In order to present a precise statement of this first step of the smoothness of $\Gamma$, let us introduce some notations. Set $\Sigma=\left\{x \in R^{3}: x_{3}=\right.$ $u(z), z \in \Omega\}, S=\left\{x \in R^{3}: x_{3}=u(z), z \in \Omega-I\right\} \subset \Sigma$, and $D=\{|\zeta|<1\}$. Let $X: D \rightarrow \Sigma$ be a uniformization (conformal representation) of the $C^{1, \lambda}$ surface $\Sigma$ with $X(0)=P \in \Gamma$, a fixed point of $\Gamma$.

THEOREM. Let $f=f_{\varepsilon}$ be a conformal mapping of $G=\{\operatorname{Im} t>0,|t|<1\}$ onto a Jordan domain $f_{\varepsilon}(G)$ containing $\{\zeta: X(\zeta) \in S,|\zeta|<\varepsilon\}$ such that $f_{\varepsilon}:(-1,1) \rightarrow X^{-1}(\Gamma)$ and $f_{\varepsilon}(0)=0$. Then there exists an $\varepsilon>0$ such that $f_{\varepsilon} \in C^{1}(\bar{G})$.

From this it is clear that the conformal representation $X\{f(t)\}$ provides, locally, a $C^{1}$ representation of $\Gamma$. The proof of the theorem relies on the strict concavity of $\psi$ and results of [2] to show that $f^{\prime} \in L^{q}(G)$ for a $q>2$.

To prove the second theorem mentioned, we assume only that $\psi \in C^{3}(\Omega)$ and is strictly concave. Hence we present a method to rectify curves determined by variational inequalities.

\section{BIBLIOGRAPHY}

1. M. Giaquinta and L. Pepe, Esistenza e regolarità per il problema dell'area minima con ostacoli in $n$ variabili, Ann. Scuola Norm. Sup. Pisa 25 (1971), 481-506.

2. D. Kinderlehrer, The coincidence set of solutions of certain variational inequalities, Arch. Rational Mech. Anal. 40 (1970/71), 231-250. MR 42 \# 6680.

3. - The regularity of the solution to a certain variational inequality, Proc. Sympos. Pure Math., vol. 23, Amer. Math. Soc., Providence, R.I., 1973.

4. Hans Lewy, On the boundary behavior of minimal surfaces, Proc. Nat. Acad. Sci. U.S.A. 37 (1951), 103-110. MR 14, 168.

5. - On minimal surfaces with partly free boundary, Comm. Pure Appl. Math. 4 (1951), 1-13. MR 14, 662.

6. H. Lewy and G. Stampacchia, On the regularity of the solution of a variational inequality, Comm. Pure Appl. Math. 22 (1969), 153-188. MR 40 \# 816.

7. - On existence and smoothness of solutions of some non-coercive variational inequalities, Arch. Rational Mech. Anal. 41 (1971), 141-253.

Scoula Normale Superiore, Pisa, Italy

School of Mathematics, University of Minnesota, Minneapolis, Minnesota 55455 